\title{
Efectos de la Vibración Aleatoria en la Vida Útil de Circuitos Impresos
}

\author{
Juan P. Cardona ${ }^{(1) *}$, John J. Leal ${ }^{(2)}$ y Juan C Vásquez ${ }^{(3)}$ \\ (1) Univ. Cooperativa de Colombia, Facultad de Ingeniería Bogotá, Dpto. de Ciencias básicas, \\ Avenida Caracas 37-63, Bogotá - Colombia (email: juan.cardonag@campusucc.edu.co) \\ (2) Univ. Nacional de Colombia, Facultad de Ingeniería, Dpto. De Ciencias básicas, Carrera 32 No. 12-00 \\ Chapinero, Via Candelaria. Palmira Valle del Cauca - Colombia. (e-mail: jlealgom@unal.edu.co). \\ (3) Universidad Pedagógica Nacional, Departamento de Tecnología, Av. Calle 72 No 11 86, Bogotá- - \\ Colombia. (e-mail: jvasquez@pedagogica.edu.co)
}

${ }^{*}$ Autor a quien debe ser dirigida la correspondencia

Recibido Ene. 14, 2016; Aceptado Mar. 11, 2016; Versión final May. 10, 2016, Publicado Dic. 2016

\begin{abstract}
Resumen
En éste artículo se determina el porcentaje teórico de disminución en horas de operación continua de la vida útil del circuito impreso sobre los componentes que estén ubicados en el punto donde la tarjeta sufre su máxima deflexión, cuando se somete a distintos niveles de vibración aleatoria. Los dispositivos electrónicos se utilizan frecuentemente bajo condiciones de vibraciones extremas y como consecuencia las conexiones de los componentes electrónicos sufren daños y el funcionamiento del dispositivo disminuye su vida útil. Para aplicar la teoría se analizó un circuito impreso de una videograbadora, instalada en la cabina de un vehículo de transporte masivo sin aislamiento de vibraciones. Se puso en marcha el vehículo y se registraron diferentes valores de vibración aleatoria con los cuales se pronostican los efectos sobre la vida útil del circuito impreso. Se concluye que la reducción en el tiempo de funcionamiento bajo estas condiciones es considerable, razón por la cual se sugiere diseñar y construir un sistema de aislamiento de vibraciones para el caso estudiado.
\end{abstract}

Palabras clave: circuito impreso; vibración aleatoria; vida útil; deflexión mecánica

\section{Effects of Random Vibration in the Useful Life of Printed Circuit}

\begin{abstract}
In this article the percentage theoric reduction in the lifetime of the printed circuit in hours of continuous operation on the components that are located at the point where the card suffers its maximum deflection is determined, when is subjected to different levels of random vibration. Electronic devices are often used under extreme vibration conditions and as a result of them connections of electronic components suffer damage and the device performance decreases its useful life. To apply the theory, a printed circuit of a video recorder, installed in the cab of a vehicle of massive transport without vibration isolation was analyzed. The vehicle was put in motion and different values of random vibration were recorded and then they are used to forecast the effects in the useful life of the printed circuit board. It is concluded that the reduction in the useful life under these conditions is considerable, so it is suggested to design and build a vibration isolation system for the case studied.
\end{abstract}

Keywords: printed circuit board; random vibration; useful life; mechanical flexing 


\section{INTRODUCCIÓN}

La vibración mecánica es el movimiento de una partícula o un cuerpo que oscila alrededor de una posición de equilibrio (Muñoz y Calderón, 2009), desde los diseños mecánicos se estudian los efectos vibratorios sobre maquinas o equipos en general, por ejemplo Zamora, Fernandes y Albizuri (2004) estudian como anticipar y controlar desajustes o fallas en los sistemas debidas a vibraciones desde su construcción, Beltrán-Carbajal (2014) establece que la vibración mecánica es bastante común en numerosas aplicaciones y problemas prácticos de sistemas de ingeniería. Las vibraciones pueden causar diversos problemas de fatiga en el material, rápido desgaste de las piezas en las máquinas, ruido excesivo, acabados pobres en productos manufacturados, aflojamiento de sujetadores, baja eficiencia de los sistemas e incluso molestias o enfermedades a las personas, según Moreno-García, Becerra-Vargas y Cárdenas-Contreras (2014) los sistemas estructurales, como los sistemas robóticos, edificios civiles, vehículos de transporte, artículos deportivos, etcétera, a menudo están sujetos a excitaciones externas e internas que provocan vibraciones no deseadas, poniendo en peligro la integridad estructural del propio sistema. Se tienen algunos antecedentes en los cuales se utilizan vibraciones como vía para caracterización de equipos, Pastoriza (2008) caracterizó equipos eléctricos utilizando medición de vibraciones, análogamente utilizaremos medidas en los equipos electrónicos para determinar niveles de vibración y así caracterizar sus fallos.

Los PCBs o circuitos impresos constan básicamente de una base aislante altamente resistente al peso, rígida, con tecnología avanzada de laminados compuestos filamentosos (tales como vidrio, boro o grafito) han tenido un aumento sustancial en su uso en las industrias aeroespacial, automotriz, ingeniería oceánica y otras (Harichandran y Naja, 1997). Sin embargo las tarjetas son sometidas a fuertes esfuerzos, tensiones y vibraciones que pueden causar daños o fallas en su funcionamiento o vida útil. Un estudio relacionado con diferentes problemas de vibraciones aleatorias que se producen en sistemas electrónicos fue presentado por Lall, Lowe y Goebel (2012) en el cual se presenta la comprensión del fenómeno de vibración en los sistemas electrónicos, aislamiento de vibración, la predicción de la duración de los puntos de soldadura en los PCB y los cálculos de confiabilidad para el correcto funcionamiento de los mismos, por otra parte Steinberg (1988) en su libro Vibration Analysis for Electronic Equipment, presenta métodos analíticos considerados en el análisis de vibración y el diseño de ensamblajes de PCBs y cajas electrónicas, en el libro se concluye que las fallas en los equipos electrónicos dependen en gran medida de la carga mecánica producidas sobre los diferentes componentes electrónicos, cables y puntos de soldadura. El autor presenta fórmulas empíricas para estimar transmisibilidad $(Q)$ de la caja electrónica y el PCB, como la siguiente:

$$
Q=C \sqrt{f_{n}}
$$

Donde $\mathrm{C}$ es una constante que tiene valores entre 0.5 y 2 dependiendo de la amplitud de excitación y $\mathrm{f}_{\mathrm{n}}$ es la frecuencia natural de vibración $(\mathrm{Hz})$ de la estructura relevante, siendo la transmisibilidad un factor adimensional. Otra ecuación presentada por Steinberg (1988) para el cálculo de la transmisibilidad (Q) se muestra en la ecuación (2) (Marks y Caterina, 2000):

$$
Q=A\left(\frac{f_{n}}{G_{i n}^{0,6}}\right)^{0,76}
$$

Donde $\mathrm{A}$ es una constante que es igual a 1 para los tipos de estructuras análogas a vigas con restricciones en sus extremos, 0.5 para placas planas, PCBs y estructuras similares con diferentes parámetros de restricción en sus extremos y 0.25 para cajas electrónicas con longitudes dos o más veces mayores que la altura y con condiciones de restricción en sus extremos, $f_{n}$ es la frecuencia natural en $\mathrm{Hz}$, y $\mathrm{G}_{\text {in }}$ es la aceleración de entrada medida en unidades de aceleración $\mathrm{G}$. Una aceleración de $1 \mathrm{G}$ es considerada como igual a la gravedad estándar que es de $9.81 \mathrm{~m} / \mathrm{s}^{2}$.

Mckeown (2013) estudió los problemas de vibración en equipos electrónicos considerando todo el sistema en tres niveles: componentes electrónicos, ensamble tarjeta y componentes y chasis o caja electrónica, revisó los métodos de análisis de vibración disponibles para cada nivel e hizo énfasis en la importancia de las pruebas de vibración modal para evitar suposiciones y simplificaciones incluidas en técnicas analíticas. También sugiere algunos enfoques útiles en modelos de elementos finitos relacionados con los componentes, placa y chasis. Los estudios realizados por Veprik y Babitsky (2000), se centraron en la respuesta dinámica del circuito impreso a fin de realizar el aislamiento de vibraciones de las cajas electrónicas. Este enfoque ha proporcionado una mejora en la protección de la caja electrónica a la vibración armónica y aleatoria en comparación con los enfoques tradicionales donde el aislamiento se basa en comprometer la amortiguación y propiedades de rigidez de los montajes, también estudiaron la placa del 
circuito impreso, diseñaron un aislador dinámico de banda ancha en miniatura y mostraron que este amortiguador puede suprimir la respuesta dinámica del sistema, teórica y experimentalmente. Esser, Huston y Miller (2003) llevaron a cabo el análisis de vibración estructural de un equipo electrónico y se obtuvieron resultados mediante el uso de modelos de análisis de elementos finitos y validación por pruebas experimentales. La figura (1) muestra que el desplazamiento dinámico máximo sufrido por un PCB bajo vibración aleatoria se produce en su centro, y en consecuencia las soldaduras de los componentes electrónicos estándar (DIP) sufren mayores daños cuando están ubicados allí.

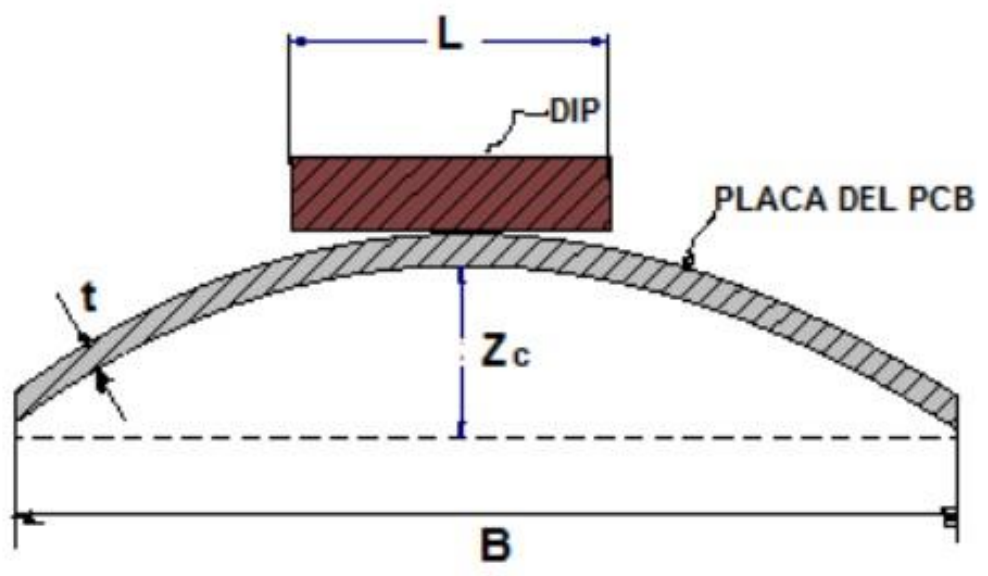

Fig.1. Desplazamiento dinámico máximo sufrido por un PCB bajo vibración aleatoria

Por otra parte Huston, Esser y Plumpton (2010) trabajaron en la patente para amortiguación de masas activas de las placas de circuitos impresos, asumiendo que la masa del PCB es despreciable, en el modelo no consideraron los distintos modos de vibración, ya que sólo estaban interesados en el primer modo de vibración del PCB, Por su parte Zampino (1995) estudió el modelado utilizando elementos finitos de una caja electrónica rectangular que contiene un circuito impreso, y logró predecir la respuesta de éste ensamble, por otra parte Cifuentes y Kalbag (1993) estudiaron la optimización de los lugares de apoyo de un PCB que influyen en los valores de la frecuencia natural del PCB, emplearon modelado por elementos finitos en sus soluciones. Así mismo, Cifuentes (1994) también llevó a cabo estudios para identificar los problemas que afectan el comportamiento dinámico del PCB cuando es sometido a vibraciones.

\section{TEORIA DE VIBRACIONES EN CIRCUITOS IMPRESOS}

Tarjeta del circuito impreso.

La tarjeta del circuito impreso se utiliza principalmente para crear una conexión entre los componentes, tales como resistencias, circuitos integrados, componentes electrónicos (DIP) y los conectores (Robertson y Plymale, 2008), por ejemplo en la figura (2a) se muestran los puntos de soldadura de componentes eléctricos, y en la figura (2b) se muestra un PCB de una tarjeta S5-31 de un equipo de video grabadora SONY.

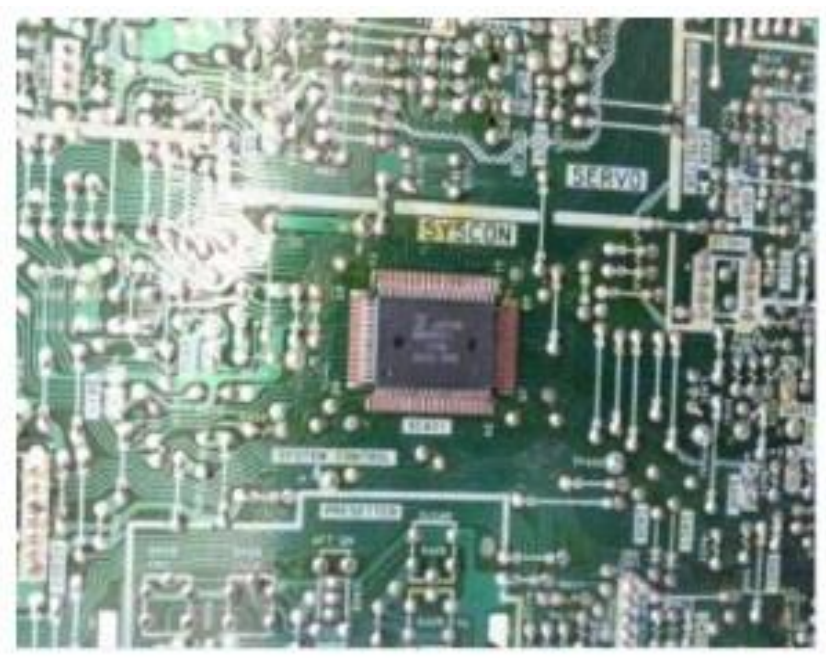

a.

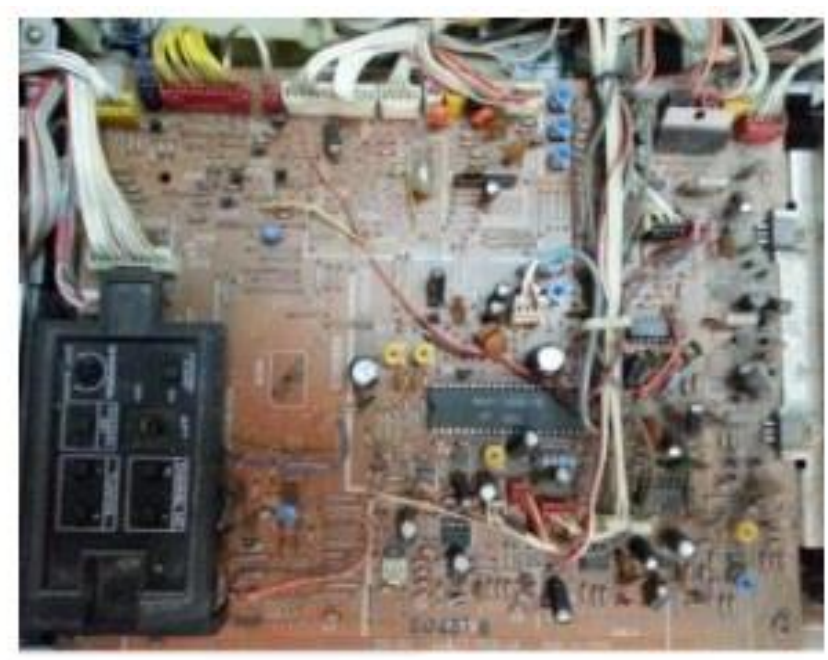

b.

Fig. 2. (a) Tarjeta de circuito impresa S5-31 del equipo de video SONY. (b) PCB del equipo de video SONY 


\section{Componentes básicos de un PCB.}

Los PCBs están compuestos normalmente de un conjunto de elementos de base como se muestra en la figura (3), dentro de estos se encuentran: La tarjeta de circuito impreso que soporta los componentes y permite interconexiones entre ellos, diversos componentes electrónicos como resistencias, DIP, capacitores y diodos, uno o más conectores que se utilizan como interfaz eléctrica entre el conjunto de la tarjeta del circuito impreso y el resto del sistema (Marks y Caterina, 2000).

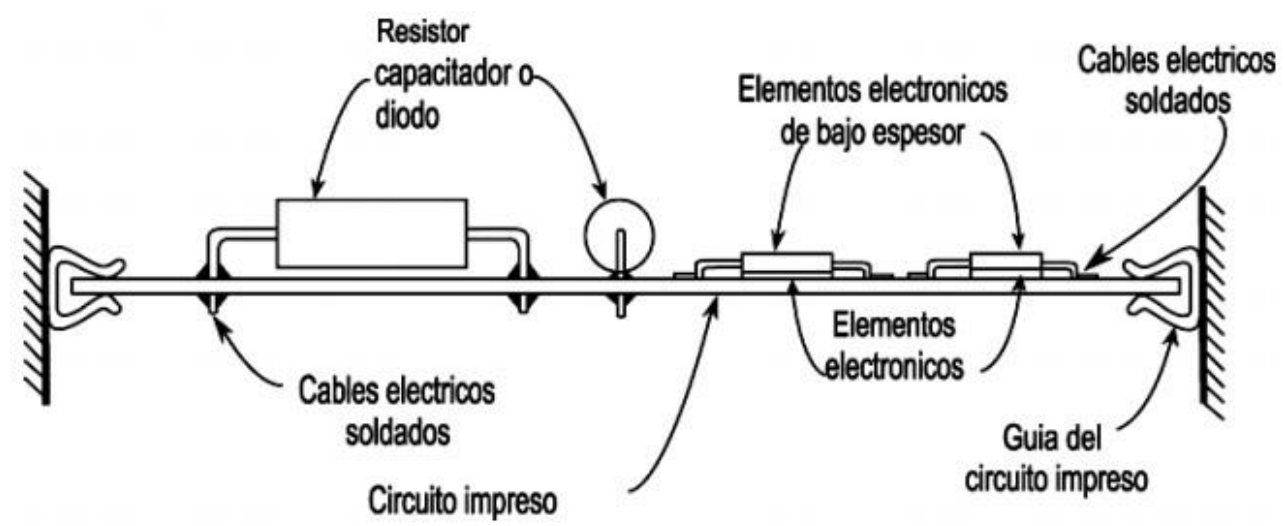

Fig. 3. Composición y montaje de un circuito impreso (PCB).

Montaje de circuitos impresos.

Los PCB se diseñan de acuerdo con las necesidades o requerimientos, tales como integridad mecánica del sistema, durabilidad contra la carga térmica, prevención de interferencias electromagnéticas y resistencia a ambientes vibratorios, para tal fin los PCB son montados en marcos o en estructuras similares a una caja denominadas cajas electrónicas que se componen de dos elementos mecánicos principales: un chasis de equipo y guías dentro del chasis como en la figura (4) lo que facilita el montaje y desmontaje.

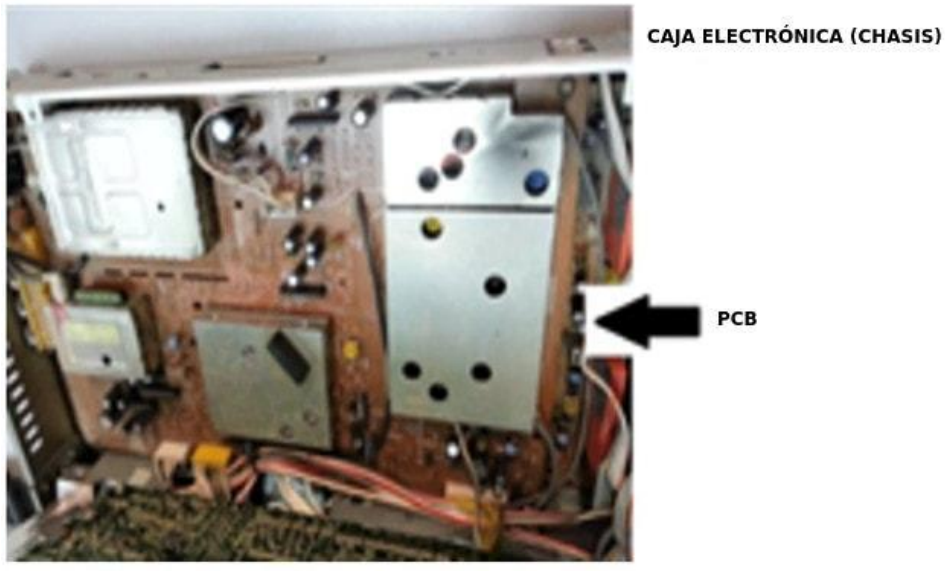

(a)

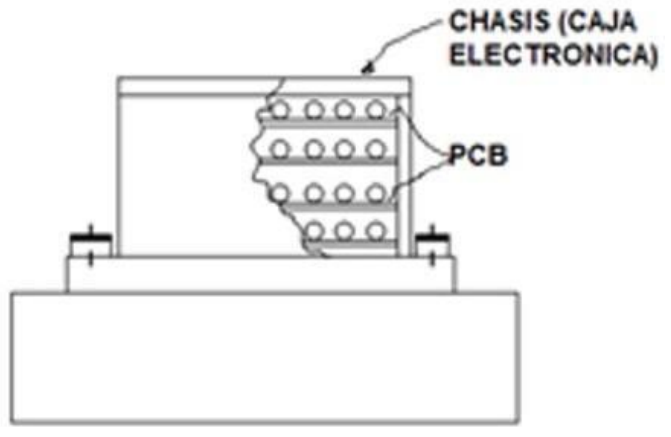

(b)

Fig. 4. a. Composición de una caja electrónica. b. Montaje de la tarjeta S5-31 del equipo de video SONY

\section{Fuentes de vibración aleatoria.}

Los equipos electrónicos, en general, están expuestos en algún momento de su vida útil, a ambientes de vibración aleatoria, ya sea por partes activas dentro del equipo en el que están instalados o como consecuencia de posibles traslados a bordo de vehículos donde se inducen fuerzas vibratorias. Estas vibraciones pueden producir muchos tipos de imperfectos que empiezan siendo leves y que con el tiempo los dañan completamente. En el caso de los equipos instalados en vehículos de transporte masivo, las fuentes de vibración son múltiples, y debido a que la mayoría de estos equipos no están diseñados para soportar los niveles producidos por el funcionamiento del motor y las imperfecciones de la carretera, se deterioran rápidamente y dejan de funcionar. 


\section{Características de la vibración aleatoria.}

Las vibraciones aleatorias ocurren en forma errática y producen un espectro continuo o lo que es lo mismo un espectro constituido por infinitas vibraciones armónicas, cada una de las cuales está caracterizada por amplitud, frecuencia y fase.

Análisis de la vibración aleatoria en el dominio del tiempo.

Dado un conjunto de datos $\left\{y_{1}, y_{2}, \ldots, y_{n}\right\}$ que representan los valores de aceleración en niveles $\mathrm{G}$ o desplazamiento en unidades de longitud de un cuerpo, para el análisis de vibraciones aleatorias, uno de los parámetros en el dominio del tiempo es el Valor Cuadrático Medio o RMS que está asociado a la potencia de la vibración, se determina de forma discreta según la ecuación (3):

$$
Y_{R M S}=\sqrt{\frac{y_{1}^{2}+y_{2}^{2}+y_{3}^{2}+y_{N}^{2}}{N}}
$$

Niveles de vibración en automóviles, camiones y trenes.

En estudios hechos según Steinberg (1988) en camiones militares, se halló que los niveles de vibración son bajos comparados con casos severos como equipos industriales, misiles, etc. En sistemas de transporte, generalmente se encuentran niveles de vibración con rangos de $2-4 \mathrm{~Hz}$ con niveles de aceleración de $0.5 \mathrm{~g}$ a $7 \mathrm{~g}$ en los amortiguadores y de 8-12 Hz y aceleración de $3 \mathrm{~g}$ en las llantas. En pruebas hechas en camiones, colocando obstrucciones a distancias variables sobre la carretera, se encontró que solo en un caso la vibración inducida fue periódica, y en todos los demás casos fue aleatoria.

\section{Fallas debidas a esfuerzos inducidos en los alambres conectores.}

Al producirse vibración se inducen esfuerzos mecánicos sobre los alambres conectores de los diferentes componentes electrónicos donde se producen la mayoría de casos de falla de los equipos electrónicos ya que los alambres conectores se rompen por fatiga debido al movimiento relativo entre el circuito impreso y los componentes electrónicos como se muestra en la figura (5).

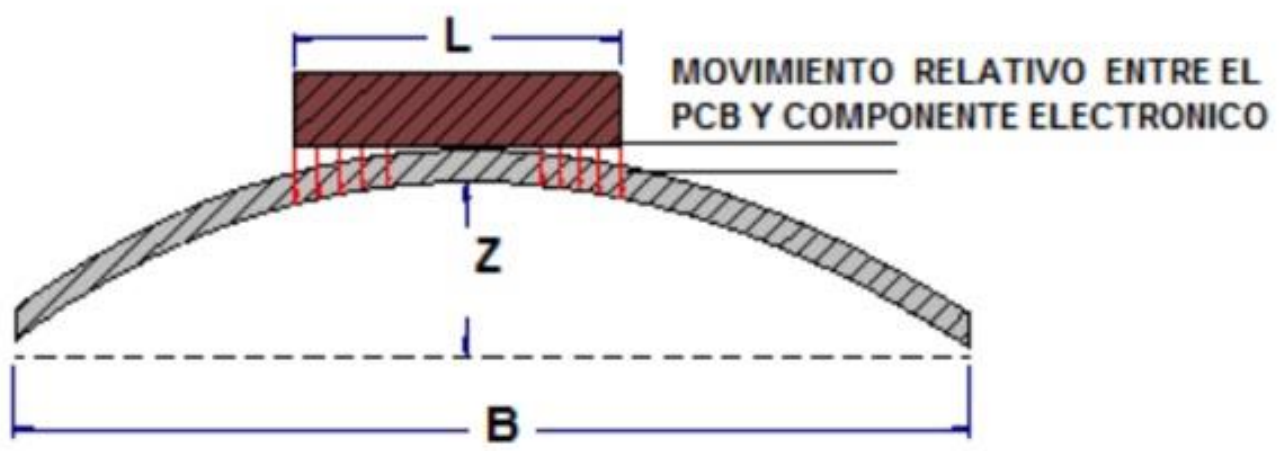

Fig. 5. Movimiento relativo entre el PCB y componente electrónico

El riesgo de ruptura es más evidente en condiciones de resonancia en este caso el componente electrónico actúa como masa y el alambre conector como resorte. Si el componente electrónico está en contacto con el circuito impreso se reduce el efecto de la resonancia sobre el sistema, pero si no lo está y el ambiente de vibración es severo, se puede esperar que los alambres conectores fallen por fatiga. Generalmente los componentes rectangulares son ubicados con sus lados paralelos a los lados del circuito impreso. Los componentes pequeños (menos de una pulgada) en raras ocasiones se afectan por ambientes de vibración. Los problemas se ocasionan generalmente sobre componentes de relativo gran tamaño (más de 2 pulgadas).

El punto crítico de ubicación de un componente electrónico en un PCB es en su parte central, porque es allí donde se presenta un cambio más rápido de curvatura. A medida que se aleja el componente de la parte central, se obtienen menores desplazamientos relativos entre el circuito impreso y el componente, reduciendo los esfuerzos inducidos en los alambres conectores y en los puntos de soldadura, incrementando su vida útil. Cuando se ubica un componente alargado paralelo a la cara más corta de un circuito impreso rectangular, se produce un cambio más rápido en la curvatura, incrementando los desplazamientos y por ende los esfuerzos inducidos sobre los alambres conectores. 


\section{Ecuaciones mecánicas para componentes electrónicos}

El desplazamiento dinámico máximo que puede sufrir un componente electrónico ubicado en el centro de una tarjeta de circuito impreso PCB, sin afectar su funcionamiento $\left(Z_{C}\right)$ está dada por la ecuación (4), que depende de los parámetros geométricos y físicos del diseño del PCB, cuando se somete a 20 millones de ciclos de esfuerzo reversible en ambientes de vibración aleatoria (Steinberg, 1988; Steinberg 2012).

$Z_{c}=\frac{0,00022 B}{c t r \sqrt{L}}$

Donde, $Z_{C}$ es el desplazamiento máximo o desplazamiento capaz del centro del PCB bajo vibración aleatoria en pulgadas, B corresponde a la longitud del borde del PCB paralelo al componente electrónico situado en el centro del tablero, medido en pulgadas, $L$ es la longitud del componente electrónico en pulgadas, $t$ es el espesor de la placa de montaje, en pulgadas, c es una constante que depende del tipo de componente analizado, así para resistencias, capacitores y diodos será de 0.75 , el valor será 1.0 para componentes electrónicos estándar (DIP) y 1.26 para componentes electrónicos con cuatro filas de pines, PGA y para componentes con pines de bronce, o con pines ensamblados en su perímetro y disipadores de calor, el valor será de 2.25 para componentes cerámicos (LCCC), el factor $r$ corresponde a la posición del componente electrónico respecto al PCB, cuando el valor de $r$ es 1 el componente electrónico se encuentra en el centro del PCB, si r tiene valor 0.707 corresponde a un componente ubicado en la mitad del lado más largo del PCB y a un cuarto del lado más corto, para $r$ igual a 0.5 , el componente electrónico se encuentra ubicado a un cuarto de cada uno de los lados.

El desplazamiento sometido $\left(Z_{S}\right)$, corresponde al desplazamiento que sufre la tarjeta PCB debido al nivel de vibración y se calcula mediante la ecuación (5): (Steinberg, 1988)

$$
Z_{s}=\frac{9,8 G_{i n} Q}{f_{n}{ }^{2}}
$$

Donde, $G_{i n}$ corresponde a la aceleración de entrada para el PCB expresada en niveles de gravedad $g, f_{n}$ es la frecuencia de resonancia o frecuencia natural de PCB medida en $\mathrm{Hz}$, y $\mathrm{Q}$ es la transmisibilidad del PCB en su frecuencia de resonancia y están relacionadas en la ecuación (1).

La aceleración RMS $G_{\text {RMS }}$ que dará lugar a una vida en fatiga de aproximadamente 20 millones de ciclos de esfuerzo reversible en un ambiente de vibración aleatoria para un PBC se calcula con la siguiente ecuación:

$$
G_{R M S}=\sqrt{\frac{\pi}{2} P f_{n} Q}
$$

Donde, $G_{\text {RMS }}$ corresponde al Nivel de aceleración Valor Medio Cuadrático o RMS soportada por el PCB, expresada en niveles de aceleración $\mathrm{g}, \mathrm{P}$ es la densidad espectral de potencia de entrada al PCB medida en $(\mathrm{in} / \mathrm{s})^{2} / \mathrm{Hz}$.

Igualando el desplazamiento capaz y sometido por medio de las ecuaciones (4) y (5) se obtiene:

$$
\frac{0,00022 B}{c t r \sqrt{L}}=\frac{9,8 G_{i n} Q}{f_{n}^{2}}
$$

La aceleración de $G$ in en la ecuación anterior es equivalente al nivel $G$ RMS de entrada y la transmisibilidad del circuito $Q$ de acuerdo con la ecuación (1), es igual a $\sqrt{ } \mathfrak{f}_{n}$, remplazando estos valores en la ecuación (7) se obtiene:

$$
\frac{0,00022 B}{c t r \sqrt{L}}=\frac{9,8 G_{R M S}\left(f_{n}\right)^{1 / 2}}{f_{n}{ }^{2}}
$$

Remplazando en la ecuación (8) el valor GRMS obtenido en (6) y el valor de transmisibilidad Q en términos de la frecuencia natural de vibración expresado en la ecuación (1) se obtiene: 


$$
\frac{0,00022 B}{c t r \sqrt{L}}=\frac{9,8 \sqrt{\frac{\pi}{2} P\left(f_{n}\right)^{1 / 2}}\left(f_{n}\right)^{1 / 2}}{f_{n}{ }^{2}}
$$

Despejando de la ecuación anterior la frecuencia natural de vibración se obtiene la fórmula para la frecuencia sometida del PCB $\left(f_{S}\right)$ para 20 millones de ciclos de esfuerzo reversible en fatiga en un ambiente de vibración aleatoria, ecuación (10):

$$
f_{s}=\left[\frac{29.4 t c \sqrt{\frac{\pi}{2} P L}}{0,00022 B}\right]^{0,8}
$$

La cual se puede expresar en términos de la aceleración $G_{\text {RMS }}$ como en la ecuación (10):

$$
f_{s}=\left[\frac{29.4 t c G_{R M S}}{0,00022 B}\right]^{0,8}
$$

\section{Cálculo de la frecuencia capaz (Frecuencia natural) del PCB.}

Según Steinberg (1988) para un PCB con la placa atornillada a dos de sus caras opuestas y las otras dos libres, se calcula la frecuencia capaz o natural de vibración mediante la ecuación (12):

$$
f_{c}=\frac{\pi}{2 B^{2}}\left[\frac{D}{\rho}\right]^{0,5}
$$

En ésta ecuación B corresponde a la longitud del borde del PCB paralelo al componente electrónico medido en pulgadas, $D$ es el factor de rigidez de la placa del PCB cuyas unidades son libras por pulgada, pes la masa por unidad de área de la placa en $\mathrm{lb} \cdot \mathrm{s}^{2} / \mathrm{in}^{3}$. En la figura (6), se muestran las características geométricas de un PCB multicapa de cobre y el polímero FR4, en la cual se aprecia la relación entre los espesores de las capas de cada material $d_{i}$ medidas en in.

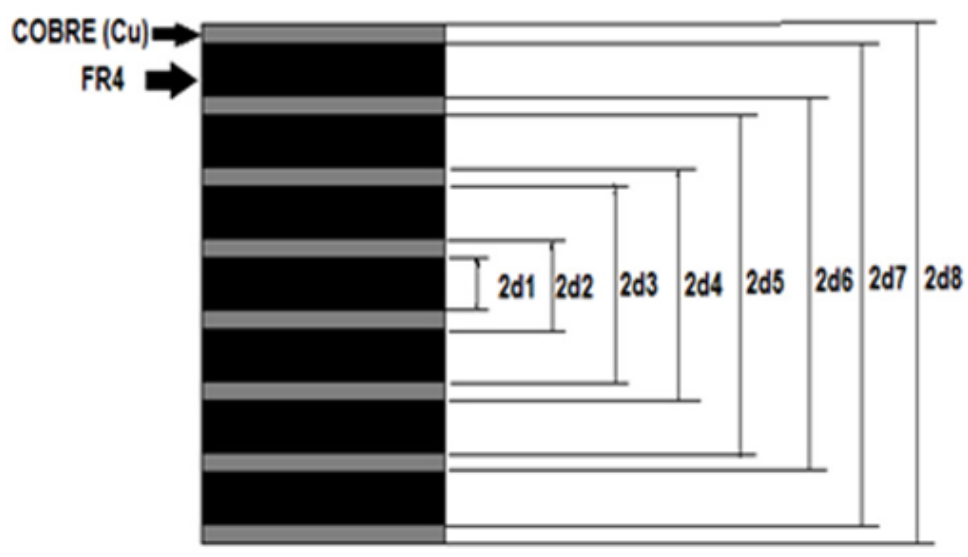

Fig. 6. Características geométricas de una tarjeta multicapa

Para hallar $\rho$ se utiliza la siguiente expresión (Blevins, 1995), $\mu_{\mathrm{i}}$ corresponde con la densidad volumétrica de cada material expresada en $\mathrm{Ib} / \mathrm{in}^{3}$.

$\rho=2\left[\begin{array}{l}\mu_{F R 4}\left(d_{1}-d_{0}\right)+\mu_{c u}\left(d_{2}-d_{1}\right)+\mu_{F R 4}\left(d_{3}-d_{2}\right)+\mu_{C U}\left(d_{4}-d_{3}\right) \ldots \\ +\mu_{F R 4}\left(d_{5}-d_{4}\right)+\mu_{C U}\left(d_{6}-d_{5}\right)+\mu_{F R 4}\left(d_{7}-d_{6}\right)+\mu_{C U}\left(d_{8}-d_{7}\right)\end{array}\right]$

El factor de rigidez $D$ cuyas unidades son lb.in del PCB está dado por la ecuación (11), donde $v$ corresponde al coeficiente de Poisson de los materiales, $\mathrm{E}_{\mathrm{i}}$ es el módulo de elasticidad de Young lb/in ${ }^{2}$ : 


$$
D=\frac{2}{3\left(1-v^{2}\right)}\left[E_{F R 4}\left(d_{1}^{3}-d_{0}^{3}\right)+E_{c u}\left(d_{2}^{3}-d_{1}^{3}\right)+E_{F R 4}\left(d_{3}^{3}-d_{2}^{3}\right)+E_{c u}\left(d_{4}^{3}-d_{3}^{3}\right)+E_{F R 4}\left(d_{5}^{3}-d_{4}^{3}\right)+E_{c u}\left(d_{6}^{3}-d_{5}^{3}\right)+E_{F R 4}\left(d_{7}^{3}-d_{6}^{3}\right)+E_{c u}\left(d_{8}^{3}-d_{7}^{3}\right)\right]
$$

\section{MATERIALES Y MÉTODOS}

Los análisis presentados hasta el momento, sirven para determinar el porcentaje en la reducción de la vida útil de un PCB sometido a cualquier ambiente de vibración aleatoria cuando su funcionamiento es en forma continua, en particular se estudió la respuesta de una tarjeta PCB de una video grabadora SONY en un ambiente de vibración aleatoria, se procedió a ubicar el equipo con su respectiva tarjeta S5-31 en la cabina de un vehículo de transporte masivo sin ningún tipo de sistema de aislamiento vibratorio ni fijación, para obtener datos reales de los niveles de vibración a los cuales se somete un PCB, al equipo se le quito la cubierta superior y se acondicionó un sistema de adquisición de datos de niveles de vibración sobre la tarjeta, el cual está constituido por un acelerómetro MNT/100 de constante estática de $100 \mathrm{mV} / \mathrm{g}$ cuyas características aparecen en la tabla (1), un osciloscopio Tectronix tds 220 para visualizar los niveles de vibración aleatoria en el tiempo. El vehículo de transporte se puso en marcha en una carretera plana con condiciones estándares y se tomaron las mediciones de vibración aleatoria al cual está sometido el PCB del equipo de video cuando el motor del vehículo se revolucionó a 750, 1000, 1500 y $2000 \mathrm{rpm}$. En la figura (7) se muestran los datos de vibración obtenidos para $1500 \mathrm{rpm}$, de tal forma que en el eje de las abscisas se representa el tiempo en segundos (s) en que se realiza la medición y en el eje de las ordenadas los niveles de tensión en milivotios $(\mathrm{mV})$.

Tabla 1. Características del acelerómetro MNT/100

\begin{tabular}{|l|l|}
\hline \multicolumn{1}{|c|}{ Característica } & \multicolumn{1}{c|}{ Rango } \\
\hline Sensibilidad estática & $100 \mathrm{mv} / \mathrm{g} 10 \%$ \\
\hline Frecuencia de respuesta & $2 \mathrm{~Hz}$ a $10 \mathrm{KHz} \mathrm{5 \%}$ \\
\hline Rango Dinámico & $80 \mathrm{~g}$ \\
\hline Rango de temperatura & -55 a $140 \mathrm{C}$ \\
\hline
\end{tabular}

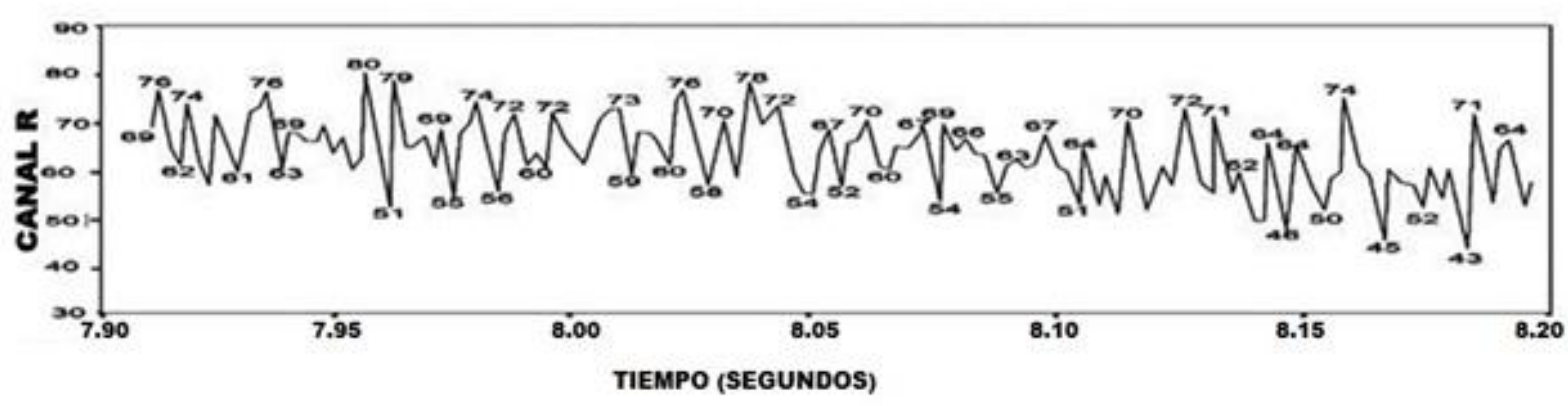

Fig. 7. Gráfica de vibración en el PCB obtenida experimentalmente con el motor girando a 1500 rpm.

\section{Caracterización del equipo de video y PCB.}

La tarjeta de video S5-31 es considerada como una placa plana multicapa constituida por ocho capas de cobre $(\mathrm{Cu})$ y siete capas de FR4 tal como se muestra en la figura (6), las propiedades geométricas de la tarjeta se muestran en la tabla (2) y en la figura (8).

Tabla 2. Características geométricas de la tarjeta S5-31 SONY

\begin{tabular}{|l|l|}
\hline Longitud del PCB (B) & 9 in \\
\hline Ancho del PCB (b) & 6 in \\
\hline 2d8 & 0,06 in \\
\hline Espesor lámina de Cobre & $1,37 \times 10^{-3} \mathrm{in}$ \\
\hline Espesor lámina de FR4 (2d1) & $7,44 \times 10^{-3} \mathrm{in}$ \\
\hline Modulo de Elasticidad del Cobre $\mathrm{E}_{\mathrm{cu}}$ & $16 \times 10^{6} \mathrm{lb} / \mathrm{in}^{2}$ \\
\hline Modulo de Elasticidad del FR4 $\mathrm{E}_{\mathrm{fr} 4}$ & $2 \times 10^{6} \mathrm{lb} / \mathrm{in}^{2}$ \\
\hline
\end{tabular}




\section{ANÁLISIS DE LA INFORMACION}

\section{Determinación de los niveles de aceleración (g) en la cabina del vehículo.}

Para determinar los niveles de aceleración experimentados por el PCB en la cabina del vehículo se traduce cada uno de los datos en milivotios $(\mathrm{mV}$ ) obtenidos del acelerómetro, a niveles de aceleración $\mathrm{g}$, utilizando la constante estática del acelerómetro $(100 \mathrm{mV} / \mathrm{g})$. De la figura (7) para $1500 \mathrm{rpm}$ los resultados se pueden visualizar en la tabla (3) para los primeros 20 datos de los 78 tomados experimentalmente, por ejemplo para el primer dato de $69 \mathrm{mV}$ el valor se divide entre la constante estática y se obtiene el nivel de vibración aleatoria al que está sometido el PCB en términos de gravedad, para éste caso $0.69 \mathrm{~g}$.

\section{Cálculos del PCB en ambiente de vibración}

Utilizando los datos de aceleración referenciados en la tabla (3), y la ecuación (3) se obtiene el valor de aceleración cuadrático $G_{R M S}=0.7 \mathrm{~g}$, utilizando éste valor, los parámetros geométricos $B=9$ in, $t=0,064$ in, $\mathrm{c}=1,26$ por el tipo de componente electrónico y la ecuación (11), se obtiene un valor de frecuencia sometida del circuito de $f_{s}=218,10 \mathrm{~Hz}$. Para hallar el valor de la transmisibilidad del circuito se utiliza la ecuación (1) y el valor de la frecuencia sometida obtenido, $Q=14,77$. A continuación calculamos la frecuencia capaz 0 natural utilizando las ecuaciones (12), (13) y (14) para obtener $\mathrm{f}_{\mathrm{c}}=50,6 \mathrm{~Hz}$.

Tabla 3. Datos experimentales tomados con el acelerómetro para vehiculó e movimiento y motor

\begin{tabular}{|c|c|c|c|c|c|}
\hline Datos & $\begin{array}{c}\text { Registro del } \\
\text { acelerómetro }(\mathrm{mV})\end{array}$ & $\begin{array}{c}\text { Niveles de } \\
\text { aceleración } \\
g\end{array}$ & Datos & $\begin{array}{c}\text { Registro del } \\
\text { acelerómetro }(\mathrm{mV})\end{array}$ & $\begin{array}{c}\text { Niveles de } \\
\text { aceleración } \\
g\end{array}$ \\
\hline 1 & 69 & 0,69 & 11 & 69 & 0,69 \\
\hline 2 & 76 & 0,76 & 12 & 63 & 0,63 \\
\hline 3 & 62 & 0,62 & 13 & 66 & 0,66 \\
\hline 4 & 74 & 0,74 & 14 & 61 & 0,61 \\
\hline 5 & 56 & 0,56 & 15 & 80 & 0,80 \\
\hline 6 & 72 & 0,72 & 16 & 54 & 0,54 \\
\hline 7 & 60 & 0,60 & 17 & 77 & 0,78 \\
\hline 8 & 75 & 0,75 & 18 & 65 & 0,65 \\
\hline 9 & 61 & 0,61 & 19 & 66 & 0,66 \\
\hline 10 & 67 & 0,67 & 20 & 65 & 0,65 \\
\hline
\end{tabular}
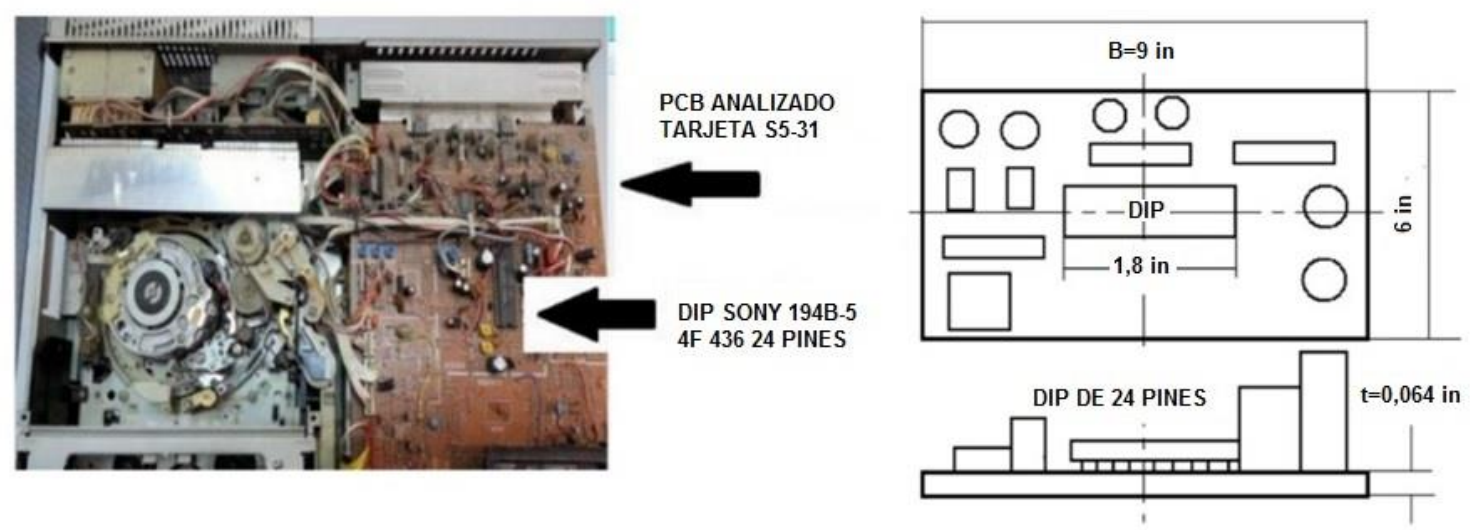

Fig. 8 Dimensiones geométricas de la tarjeta s5-31 SONY

Se calcula el desplazamiento sometido del PCB utilizando la ecuación (5) para obtener $2,1 \times 10^{-3}$ in, correspondiente al desplazamiento dinámico máximo que sufre la tarjeta cuando se somete a una frecuencia de $218,1 \mathrm{~Hz}$. Por otra parte se calcula el desplazamiento capaz o natural del PCB en ausencia de vibración utilizando la ecuación (4) para obtener 0.02in de desplazamiento. Al comparar los desplazamientos sometido y capaz, es mayor el desplazamiento capaz lo que indica que el PCB no fallará inmediatamente al someterse al ambiente de vibración promedio de $0.7 \mathrm{~g}$, es decir que el fallo no será instantáneo. En la figura (9) se muestra la deformación máxima que sufre la placa del PCB cuando está vibrando a su frecuencia natural $(50,6 \mathrm{~Hz})$, dicha simulación se realizó con el software ANSYS15, utilizando el elemento estructural SHELL 99 apropiado para simular placas planas de materiales compuestos, la 
placa se supuso sujeta en los cuatro nodos de las esquinas para simular la condición de sujeción con tornillos en sus cuatro esquinas se observa que la deformación máxima y los esfuerzos máximos se presentan en el centro de la placa.

\section{RESULTADOS Y DISCUSIÓN}

Para establecer el porcentaje de reducción de vida en horas como consecuencia de la exposición del PCB al ambiente de vibración aleatoria a la que está sometido, se calculan las horas que funcionará continuamente sin sufrir daños a 20 millones de ciclos y una frecuencia natural (En ausencia de vibración) de $50,6 \mathrm{~Hz}$ utilizando el siguiente calculo:

VIDA $($ Horas $)=\frac{20 \times 10^{6} \mathrm{ciclos}}{(50,6 \mathrm{ciclos} / \mathrm{sec})(3600 \mathrm{sec} / \text { hora })}=109.79$
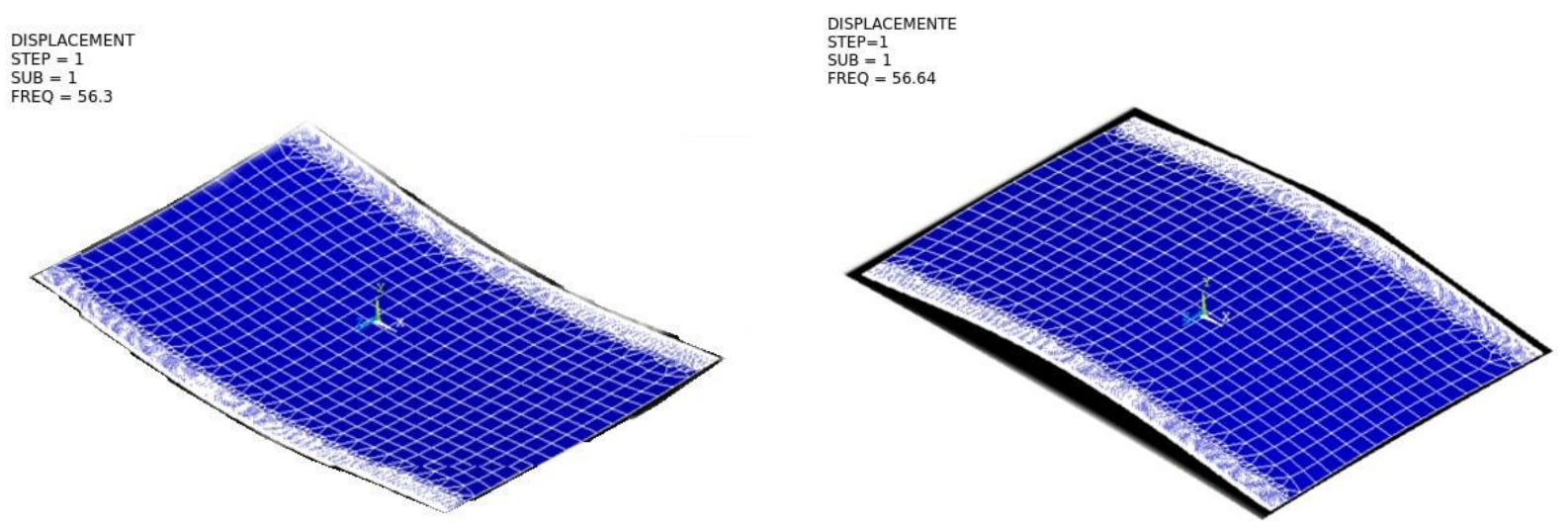

Fig. 9. Simulación de la deformación máxima que sufre la placa del PCB cuando vibra a su frecuencia natural

De la misma manera se hace el cálculo utilizando la frecuencia sometida $f_{\mathrm{S}}=218.1 \mathrm{~Hz}$, se obtiene el número de horas 25,47 horas de funcionamiento continuo para $1500 \mathrm{rpm}$ del motor, lo que quiere decir que se produciría una disminución en la vida útil del PCB del 74,7\%. De igual forma se realizan los cálculos para los otros niveles de vibración y los resultados se muestran en la tabla (5).

Tabla 5. Disminución de vida útil en horas continúas de funcionamiento del PCB a diferentes niveles de vibración

\begin{tabular}{|c|c|c|c|c|}
\hline $\begin{array}{c}\text { RPM del } \\
\text { motor }\end{array}$ & G $_{\text {RMS }}$ & $\begin{array}{c}\text { Frecuencia de vibración del } \\
\text { PCB }(\mathrm{Hz})\end{array}$ & $\begin{array}{c}\text { Vida útil del PCB } \\
\text { (horas) }\end{array}$ & $\begin{array}{c}\text { Porcentaje de disminución } \\
\text { de vida }\end{array}$ \\
\hline 0 & 0 & 50,6 & 109,79 & $0 \%$ \\
\hline 750 & 0,92 & 271.40 & 20.46 & $79,7 \%$ \\
\hline 1000 & 0,8 & 242.69 & 22.89 & $77,3 \%$ \\
\hline 1500 & 0,7 & 218.12 & 25.47 & $74,7 \%$ \\
\hline 2000 & 0,6 & 192.8 & 28.81 & $71,4 \%$ \\
\hline
\end{tabular}

\section{RECOMENDACIONES}

Los componentes mecánicos o de unión (soldaduras) de las tarjetas electrónicas se diseñan utilizando teoría de fallas para ambientes de vibración periódica o estática, sin embargo los dispositivos se exponen a otro tipo de ambientes que generan esfuerzos mayores y por tanto disminuyen su vida útil generando fallos. Es necesario tener en cuenta éste aspecto para hacer el análisis del diseño del componente electrónico en éste tipo de ambiente, bien sea cambiando las dimensiones o el tipo de soldadura o utilizando sistemas de aislamiento propios para los ambientes vibratorios.

\section{CONCLUSIONES}

La vida útil de una tarjeta impresa se reduce notablemente cuando es sometida a una vibración aleatoria durante una exposición continua, en comparación con el número de horas para el cual fue diseñado en un ambiente sin vibraciones. Se espera que el número de horas de vida útil sea mucho mayor, cuando la exposición no sea continua sino durante periodos cortos de tiempo, pero esto hace parte de otra investigación respecto al tema por la fatiga de los materiales. 


\section{REFERENCIAS}

Beltrán-Carbajal, F., Variable frequency harmonic vibration suppression using active vibration absorption. Revista Facultad de Ingeniería Universidad de Antioquia, ISSN 0120-6230 (En línea), 73, 144156. (2014) https://aprendeenlinea.udea.edu.co/revistas/index.php/ingenieria/article/view/18126/17651, Acceso: Mayo 8 (2016)

Blevins. R.D., Formulas for Natural Frecuency and Mode Shape, 1a. Edición. Krieger Publishing Company. (1995)

Cifuentes, A.O., Kalbag, A., Dynamic behavior of printed wiring boards: increasing board stiffness by optimizing support locations. Doi: 10.1109/ECTC.1993.346830, Electronic Components and Technology Conference, 1993. Proceedings., 43rd , pp.270-275, 1-4 Jun. (1993)

Cifuentes, A. O., Estimating the dynamic behavior of printed circuit boards. Components, Packaging, and Manufacturing Technology, Part B: Advanced Packaging, Doi: 10.1109/96.296433, IEEE Transactions on, 17(1), 69-75. (1994)

Esser, B., Huston, D. R., y Miller, J., Aerospace electronics weight reduction through the use of active mass damping. In Smart Structures and Materials. Doi: 10.1117/12.484752, International Society for Optics and Photonics. 5052, 433-444. (2003)

Esser, B., Huston, D., Active Mass Damping of Electronic Circuit Boards, Doi:10.1016/j.jsv.2003.11.055 Journal of Sound and Vibration, 277(1-2), 419-428, (En línea) (2004)

Harichandran, R. S., y Naja, M. K., Random vibration of laminated composite plates with material nonlinearity. International journal of non-linear mechanics, Doi: 10.1016/S0020-7462(96)00089-3, 32(4), 707720. (Por suscripción) (1997)

Huston, D. R., Esser, B., y Plumpton, J. O.. U.S. Patent No. 7,765,032. Washington, DC: U.S. Patent and Trademark Office. (2010)

Ho, V.C., Veprik, A.M., Babitsky. V.I., Ruggedizing Printed Circuit Boards Using a Wideband Dynamic Absorber, Shock and Vibration. Doi: 10.1155/2003/185798, 10, 195-210. (2013)

Jung H., Park T., Seo J., Han S., Kim S., Structural Vibration Analysis of Electronic Equipment for Satellite under Launch Environment, Doi: 10.4028/www.scientific.net/KEM.270-273.1440, Key Engineering Materials, 270-273 pp.1440-1445. (2010)

Lall, P., Lowe, R., Goebel, K., Prognostics Health Management of Electronic Systems Under Mechanical Shock and Vibration Using Kalman Filter Models and Metrics, DOI: 10.1109/TIE.2012.2183834, Industrial Electronics, IEEE Transactions on, 59(11), pp.4301-4314, (2012)

Marks L., J. Caterina. A., Printed Circuit Board Assembly Design, ISBN: 9780070411074, McGraw-Hill. (2000)

McKeown. Mechanical Analysis of Electronic Packaging Systems, ISBN-13: 978-0824770334, Marcel Dekker, Inc. (2013)

Moreno-García, F. E., Becerra-Vargas, J. A., y Cárdenas-Contreras, E. M.. Implementación de una estrategia fuzzy adaptativa para el control activo de vigas vibratorias. Doi: 10.19053/01211129.2711 Revista Facultad de Ingeniería (Fac. Ing.), 23(36), 53-64. (2014)

Muñoz, M. R. P., \& Calderón, W. R. Modelación del fenómeno de vibración forzada formulando una analogía eléctrica con el programa de elementos finitos,

Ansys. http://www.revistas.unal.edu.co/index.php/ingeinv/article/view/15137/15931, ISSN: 0120-5609, Ingeniería e Investigación, 29(1), 5-12. (2009)

Pastoriza, V., Poza, F., Marino, P., Y Machado, F., Extracción de Características en Equipos Eléctricos basada en Medida de Vibraciones. doi:10.1612/inf.tecnol.3947it.07, Inf. tecnol. 19(4), pp. 71-78. (2008)

Robertson, W. H., y Plymale, J. M.. U.S. Patent No. 7,353,467. Washington, DC: U.S. Patent and Trademark Office. (2008) 
Robertson. C. R. Printed Circuit Board Designers, Reference: Basics, ISBN: 0130674818 Prentice Hall. (2008)

Steinberg. D. S.. Preventing Thermal Cycling and Vibration Failures in Electronic Equipment, ISBN: 9780471357292 John Wiley \& Sons, Inc. (2012)

Steinberg, Dave S. Vibration analysis for electronic equipment. ISBN: 978-0-471-37685-9, New York, WileyInterscience, 460 p. No individual items are abstracted in this volume. 1 (1988)

Veprik, A. M.; Babitsky, V. I.. Vibration protection of sensitive electronic equipment from harsh harmonic vibration. Doi:10.1006/jsvi.2000.3098, Journal of Sound and Vibration, 238(1), p. 19-30. (2000)

Veprik. A. M., Vibration Protection of Critical Components of Electronic Equipment in Harsh Environmental Conditions, Journal of Sound and Vibration, 259 (1), p.161-175. (2014)

Zampino, M.A., Vibration analysis of an electronic enclosure using finite element analysis, Doi: 10.1109/SOUTHC.1995.516125, Southcon/95. Conference Record , pp.328-333, 7-9 Mar. (1995)

Zamora, E., Fernandes, M. H., y Albizuri, J., Caracterización Dinámica de Máquinas Herramienta y Diseño de un Sistema Activo de Reducción de Vibraciones durante el Mecanizado. Doi: 10.4067/S071807642004000100004, Información tecnológica, 15(1), 23-28. (2004) 